\title{
Evaluation of a possible association between a history of dentoalveolar injury and the shape and size of the nasopalatine canal
}

\author{
Valerie G. A. Suter ${ }^{1}$ - Reinhilde Jacobs ${ }^{2}$ - Marcia R. Brücker ${ }^{3}$ - Alberto Furher ${ }^{2}$. \\ Jim Frank ${ }^{2,4}$. Thomas von Arx ${ }^{1}$ - Michael M. Bornstein ${ }^{1,2}$
}

Received: 22 January 2015 / Accepted: 21 July 2015 / Published online: 7 August 2015

(C) Springer-Verlag Berlin Heidelberg 2015

\begin{abstract}
Objectives Maxillary incisors (MI) are often affected by dentoalveolar injury resulting in tooth devitalization and apical periodontitis. The aim of the present study was to analyze any association between a history of dentoalveolar injury and the shape and size of the nasopalatine canal (NC) using cone beam computed tomography (CBCT).

Material and methods Patients were allocated to the trauma group if they had a history of dentoalveolar injury and a root filling in at least one MI and/or one missing MI. As controls, 100 matched-controlled (age and gender) patients were selected. NC dimensions including length, width at midway, and diameter of incisal and nasal foramen were measured in sagittal and axial CBCT planes. Furthermore, an evaluation of NC bulging signs, apical osteolysis of MI, and its fusion with $\mathrm{NC}$ was performed. Results In the trauma group $(n=96), 31.3 \%$ had at least one missing MI, and $95.8 \%$ had a root filling in a MI. The anteroposterior dimension of the incisive foramen $(p=0.02)$ and of the $\mathrm{NC}$ at midway $(p=0.04)$ was significantly larger in the
\end{abstract}

Michael M. Bornstein

michael.bornstein@zmk.unibe.ch

1 Department of Oral Surgery and Stomatology, School of Dental Medicine, University of Bern, Freiburgstrasse 7, 3010 Bern, Switzerland

2 OMFS IMPATH Research Group, Department of Imaging and Pathology, Faculty of Medicine, University of Leuven, Leuven, Belgium

3 Radiologic Center, School of Dentistry, Pontifical Catholic University, Rio Grande do Sul Porto Alegre, Brazil

4 Department of Oral Health Sciences, Faculty of Medicine, University of Leuven, Leuven, Belgium trauma group. Significantly more cases with a bulging sign were found in the trauma $(n=19)$ than in the control group $(n=$ $3, p=0.001)$. An apical osteolysis was identified in $5.1 \%$ of MI, and 12/38 did show a fusion with the NC.

Conclusion Wider dimensions of the NC and a bulging sign may suggest a former dentoalveolar injury to the anterior maxilla. Periapical osteolysis of central MI over $5 \mathrm{~mm}$ in diameter tends to fuse with the NC.

Clinical relevance In patients with a history of dentoalveolar injury and/or apical periodontitis, the NC should be evaluated on available CBCT images. Any inflammatory processes in the neighboring teeth should be recognized and eliminated as they may initiate bulging of the $\mathrm{NC}$ and/or the formation of a nasopalatine duct cyst (NPDC). NC with bulging signs should be monitored clinically and radiographically to diagnose a NPDC in an early stage.

Keywords Anterior maxilla $\cdot$ Nasopalatine canal $\cdot$ Incisive canal $\cdot$ Incisive foramen $\cdot$ Periapical lesion $\cdot$ Cone beam computed tomography $\cdot$ Dentoalveolar injury

\section{Introduction}

A maxillary incisor trauma is evidenced in one out of four adults [1]. Main complications after dentoalveolar injury include tooth devitalization and root resorptions (inflammatory or replacement) $[2,3]$. Thus, treatment following trauma may involve replacement of missing or hopeless teeth and endodontic and reconstructive interventions $[4,5]$. If necrosis is not diagnosed, an apical periodontitis may develop. A direct consequence of the inflammatory process is a progressive periapical osteolysis.

The nasopalatine canal (NC) is an important anatomical structure of the anterior maxilla, located in the midline and posterior to the maxillary incisors (MI). The incisive foramen 
is the palatal opening of the $\mathrm{NC}$ and is located underneath the mucosal incisive papilla. The $\mathrm{NC}$ contains the nasopalatine (incisive) nerve, the terminal branches of the nasopalatine artery, and the veins from the anterior palate forming a neurovascular bundle surrounded by connective tissue, fat, and seromucous glands $[6,7]$. In the last decade, the introduction and widespread use of cone beam computed tomography $(\mathrm{CBCT})$ in dental medicine enabled detailed research of anatomical structures in the orofacial region on larger patient series [8-10]. Thus, different anatomical variations of the NC were described in the literature. Mraiwa et al. [11] reported for the first time anatomical details regarding variations of the $\mathrm{NC}$ morphology, and Bornstein et al. [12] classified the NC in three different types: the single canal (45\% incidence), two separate parallel canals ( $15 \%$ incidence), and a $\mathrm{Y}$ type of canal with two or more nasal openings ( $40 \%$ incidence). Other studies suggested more subclassifications, depending on the number of nasal openings (foramina of Stensen) $[6,7,11,13]$ and/or did not distinguish the $Y$ type from two parallel canals $[6,7,14]$.

Due to the proximity of the $\mathrm{NC}$ to the roots of the central incisors, structures within the NC may react biologically in case of displacements (following dentoalveolar injury) or infection of these teeth. It has been shown that inflammatory cytokines such as IL-1, IL-6, and TNF stimulate epithelial cell proliferation and may play a role in the pathogenesis of cyst formation [15]. The exact cause and pathogenesis of nasopalatine duct cysts (NPDC) still remain controversial. Some authors suggest a spontaneous proliferation of epithelial remnants located within the NC $[16,17]$. Furthermore, a stimulation to the epithelial remnants due to localized trauma or infections has been proposed in the literature as possible etiological factor $[18,19]$. Based on immunohistochemical profiles of the lining epithelia, a recent study hypothesized that NPDC may derive from the epithelial rest of Malassez and thus are directly associated with periapical periodontitis [20].

The aim of the present study was to look for any association between a history of dentoalveolar injury and the shape and size of the NC. Furthermore, an analysis of the presence/ absence of any periapical osteolyses and their fusion with the $\mathrm{NC}$ was performed.

\section{Materials and methods}

\section{Study sample and inclusion and exclusion criteria}

Patients referred for CBCT scanning performed at the Oral Imaging Center, University Hospitals Leuven (Belgium) between July 2008 and December 2012 were potentially eligible for the study. All patients had given their informed consent for the CBCT. From this database, CBCT scans of patients $(n=$ 100; mean age 46 years, range 9 to 85 years) with a known history of dentoalveolar injury to the anterior maxilla dating back at least 12 months before imaging were consecutively selected for this retrospective analysis. Thus, the CBCT protocols for this study including the respective fields of view were not specifically selected for the previous dentoalveolar injury. Furthermore, 200 patients (mean age 37 years, range 7 to 82 years) without known dentoalveolar injury who had CBCT imaging performed on the anterior maxilla between July 2011 and December 2012 were consecutively selected from the files. Inclusion criteria for all CBCT scans were that the anterior maxilla was entirely represented on the image and that no artifact was disturbing their interpretation. Patients with any congenital deformities or syndromes affecting the orofacial region were excluded. Furthermore, patients who had received extended maxillofacial surgery, i.e., due to jaw fractures, were also excluded. The study followed the guidelines of the declaration of Helsinki. The study protocol was submitted to and reviewed by the local ethical committee of the University Hospitals Leuven (S57587) but was exempt from formal approval due to its retrospective nature.

\section{Radiological analysis}

CBCT images were taken with the Scanora 3D (Soredex, Tuusula, Finland) using a field of view of $7.5 \times 10,7.5 \times 14.5$, or $13 \times 14.5 \mathrm{~cm}$, voxel size of $0.25 \mathrm{~mm}$, voltage of $85 \mathrm{kV}$, and exposure setting of $30 \mathrm{mAs}$ or with the 3D Accuitomo 170 (Morita, Kyoto, Japan) with a field of view of $6 \times 6,8 \times 8$, or $5 \times 10 \mathrm{~cm}$, voxel size of $0.125 \mathrm{~mm}$, voltage of $90 \mathrm{kV}$, and exposure setting of $87.5 \mathrm{mAs}$. CBCT images were observed in the IMPAX software (Agfa, Mortsel, Belgium) in a dimmed room with ambient light conditions at a $60-\mathrm{cm}$ distance from a 20-in. medical display (MDRC-2120, Barco NV, Kortrijk, Belgium). The two observers (A.F. and J.F.) were trained, calibrated, and supervised by two professors in dentomaxillofacial radiology (M.R.B. and R.J.). Critical cases were discussed and referred to the expert consensus of both professors.

The data were reconstructed with slices at an interval of $0.5 \mathrm{~mm}$. Then, the CBCT slices were adjusted to place the NC in a vertical position in the coronal views and the palate/floor of the nose in a horizontal position in the sagittal view. The anatomic type of canal was evaluated in all planes and allocated to one of the three groups described by Bornstein and coworkers [12]. Measurements of $\mathrm{NC}$ were performed in sagittal (Fig. 1) and axial CBCT planes. In the sagittal plane, the antero-posterior diameter of the nasal foramen (Stensen) (no. 1), of the incisive foramen (no. 2), and of the $\mathrm{NC}$ at midway (no. 3) and the $\mathrm{NC}$ length (no. 4) were measured. If $\mathrm{NC}$ was of type $\mathrm{B}$ (two separate parallel canals) and type $\mathrm{C}$ (Y type of canal with two or more nasal openings), the lengths and diameters of the different canals and/or openings were added together and a mean value calculated. In the axial plane, the mesio-distal diameter of the incisive foramen (no. 5) and the $\mathrm{NC}$ at midway (no. 6) were measured, and similarly when the $\mathrm{NC}$ was a type $\mathrm{B}$, measurements were 


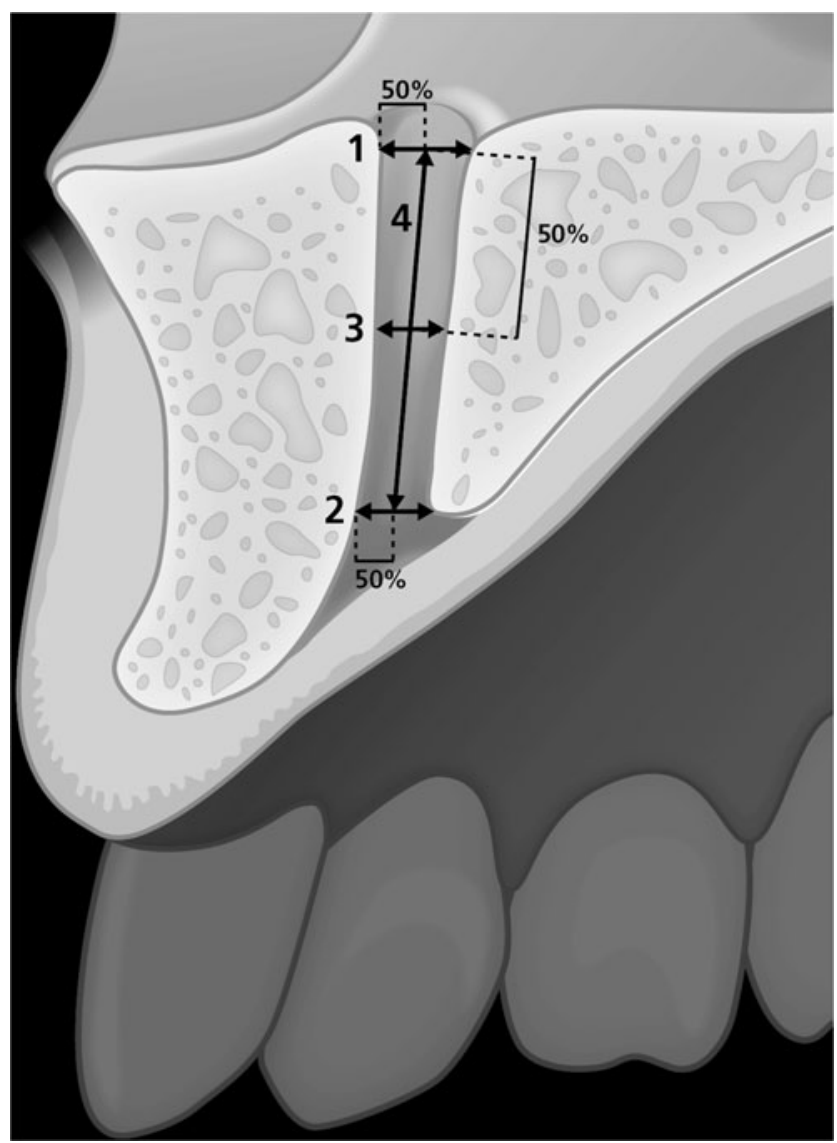

Fig. 1 Schematic view of the different measurements performed on the sagittal planes of the CBCT images (no. 1 to no. 4). The measurements in the axial plane are not shown

added and an average was calculated. Furthermore, all NC were evaluated for any bulging signs (Figs. 2 and 3). If present, the antero-posterior dimension of the bulging area was measured in the sagittal plane, the mesio-distal dimension in the axial plane, and the cranio-caudal dimension in the coronal plane. Central and lateral incisor teeth were recorded as present or missing and evaluated for the presence/absence of a root canal filling. As well, the presence of a periapical osteolysis was identified for each maxillary incisor, and it was evaluated, if there was a fusion with the NC (Figs. 4 and 5). The bucco-palatal, mesio-distal, and craniocaudal dimensions of the apical or fused osteolysis were measured. Osteolytic areas in edentulous sites and around dental implants were also recorded and measured identically. Due to the different shapes of osteolytic lesions, the highest measured value of each dimension was considered for further analysis.

\section{Group allocation and analysis}

Cases were allocated to the trauma group, if they had a positive history of dentoalveolar injury $(n=100)$ and a root canal filling in at least one MI and/or at least one missing MI or replaced by an implant/transplant. Cases were allocated to the control group if they had no root canal filling in any MI, no

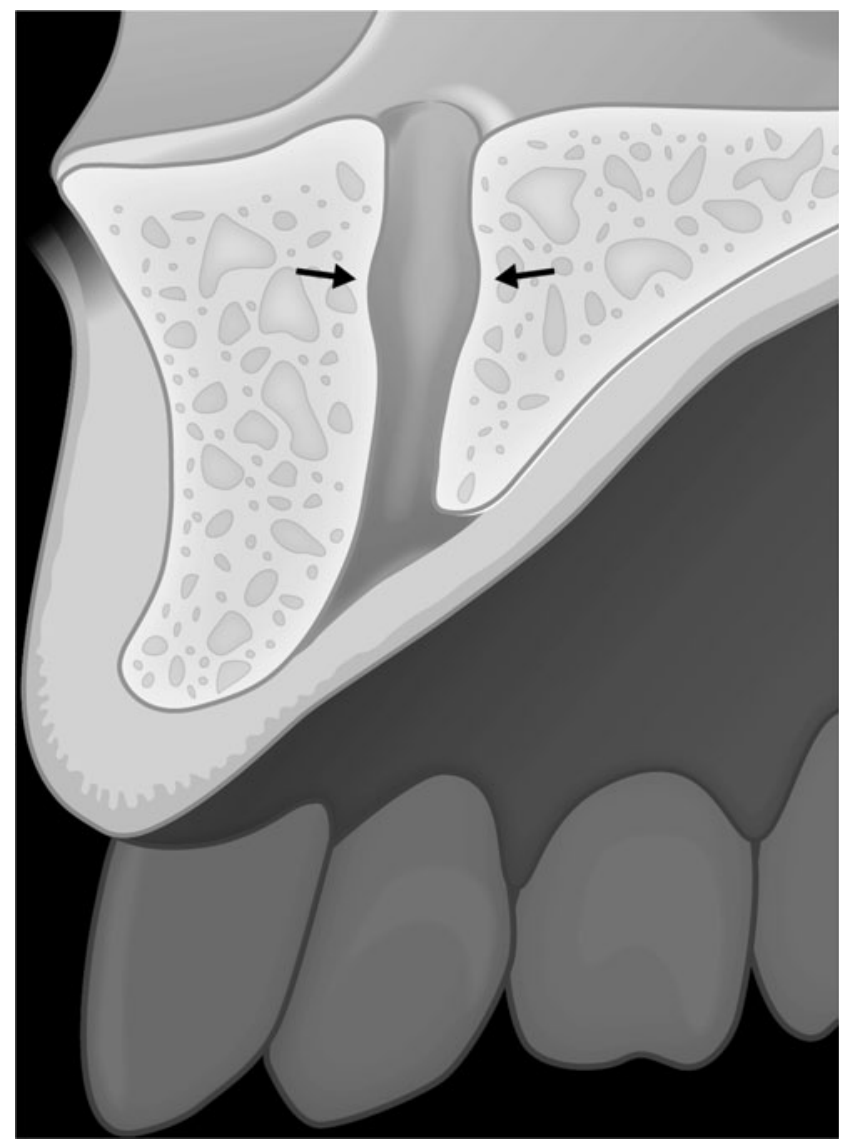

Fig. 2 Schematic view of a typical bulging sign exhibiting a local enlargement (arrows) of the nasopalatine canal

missing MI, and no trauma history. All cases without history of dentoalveolar injury but having at least one missing MI and/or a root canal filling in one MI were not considered for further analysis. Among the initial data of 200 cases without known dentoalveolar injury, 148 were fulfilling these criteria. For further analysis, an age- and gender-matched controlled selection to the trauma group was performed resulting in 100 patients for inclusion in the control group.

\section{Statistical analysis}

All data were first analyzed descriptively. For comparison between the two groups (dentoalveolar injury and control), the dimensions of the NC (no. 1 to no. 6) and of its bulging were analyzed using exact Wilcoxon rank-sum tests. Comparison of the frequency of bulging signs between both groups was assessed with Fisher's exact test. The binominal test was used to compare the distribution of patients/teeth between the groups with and without fusion of osteolytic lesions with the NC. The measurements of the dimensions of the osteolytic lesions were compared using Wilcoxon rank-sum tests between these two groups (e.g., with and without fusion with the NC).

All analyses were performed with the statistical software R (Version 3.0.1 for Windows, Institute for Statistics and 


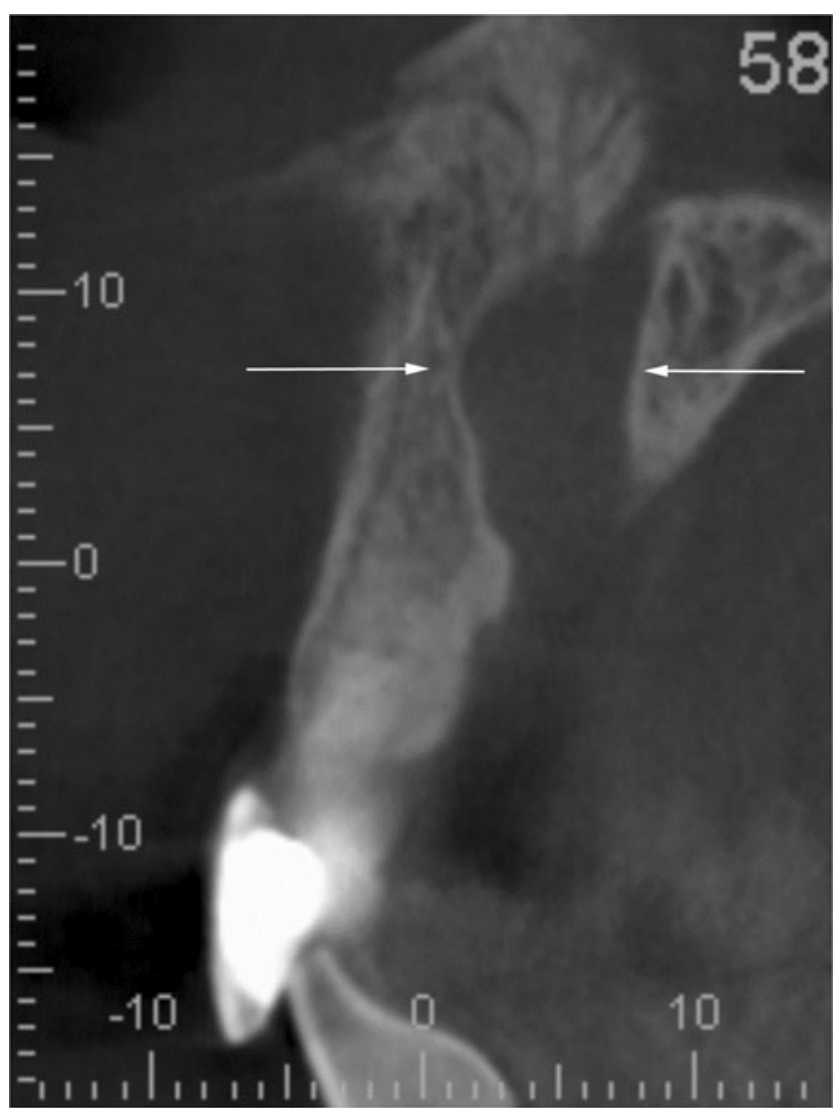

Fig. 3 Representative CBCT image (Accuitomo 170, field of view of $6 \times$ $6 \mathrm{~cm}$, voxel size $0.125 \mathrm{~mm}$ ) in the sagittal plane illustrating a nasopalatine canal with a remarkable enlargement, identified as bulging sign (arrows)

Mathematics of the WU Wien, Vienna, Austria; http://www. R-project.org). Due to the explorative nature of the study, no correction has been applied for multiple testing. Significance levels were set at $p \leq 0.05$.

\section{Results}

Of the 100 patients with positive history of dentoalveolar injury, four were excluded for further analysis after examination of the CBCT, because of the following reasons: history of dentoalveolar injury without any missing MI or root canal filling in MI $(n=2)$, palatoschisis $(n=1)$, and surgically removed NPDC $(n=1)$. The demographic distribution of the 96 patients of the dentoalveolar injury group and the 100 patients of the control group are presented in Table 1 . In the trauma group, $31.3 \%$ had at least one missing MI and $95.8 \%$ had a root filling in a MI. In total, 40 MI were missing and 160 MI were root canal filled. More central MI $(n=108)$ than lateral MI $(n=52)$ presented with a root canal filling (Table 1$)$. Regarding the type of $\mathrm{NC}$, the $\mathrm{Y}$ type was most frequently found, followed by the single $\mathrm{NC}$, while only few cases had two parallel canals (Table 1$)$. In most NC (54.5\%), two nasal openings were identified, while in $39.3 \%$ only one, and in

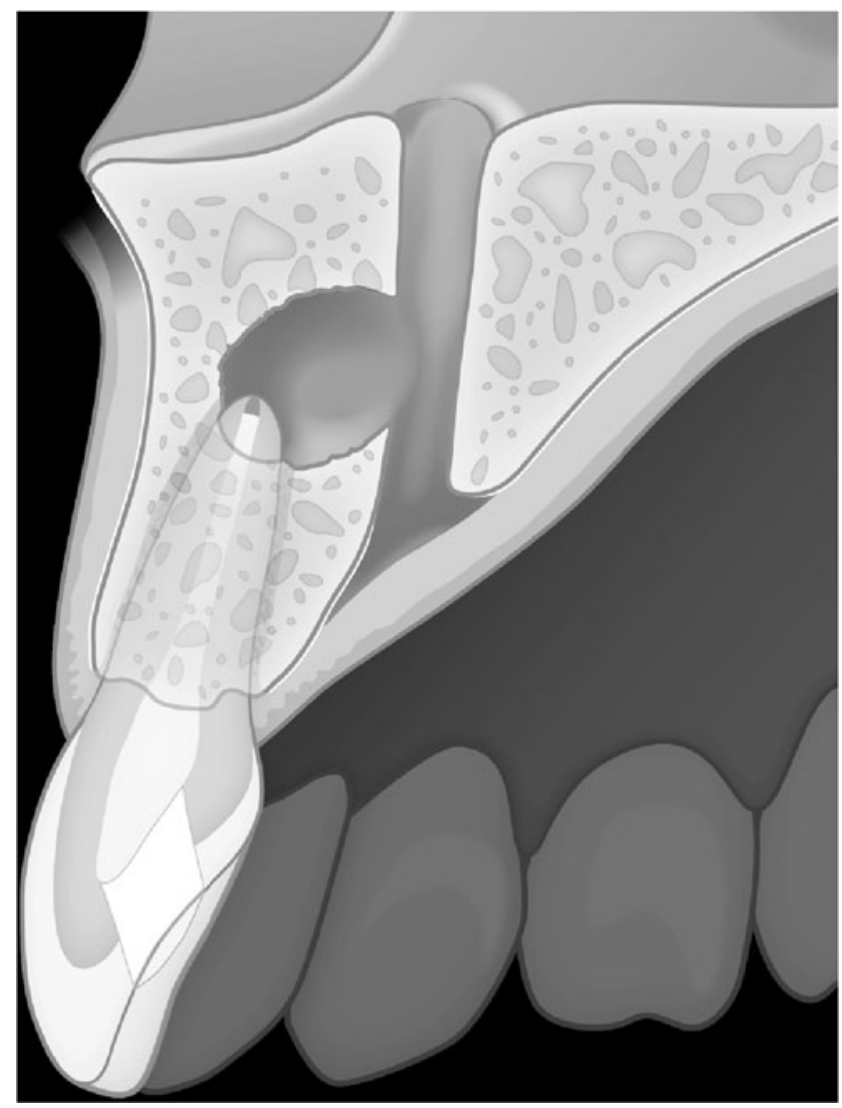

Fig. 4 Schematic illustration of an osteolytic lesion apical of the root of a maxillary incisor showing fusion with the nasopalatine canal

$6.1 \%$ three or more were seen. The type of canal and the number of nasal openings were distributed equally between the trauma and the control group.

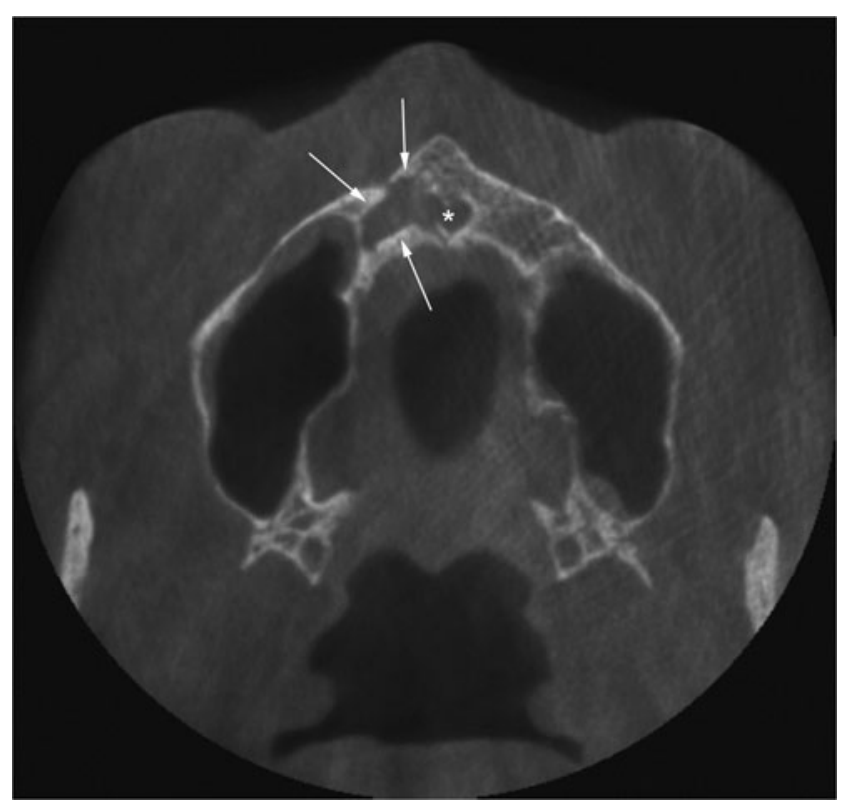

Fig. 5 Osteolytic lesion of irregular shape (arrows) in the axial view of a CBCT image (Scanora 3D, field of view $13 \times 14.5 \mathrm{~cm}$, voxel size of $0.25 \mathrm{~mm}$ ), which has fused with the nasopalatine canal (asterisk) 
Table 1 Distribution of the included patients regarding age, gender, maxillary incisors, endodontic fillings, and type of the nasopalatine canal

\begin{tabular}{|c|c|c|c|}
\hline & All & Dentoalveolar injury group & Control group \\
\hline Patients $(n)$ & 196 & 96 & 100 \\
\hline \multicolumn{4}{|l|}{ Gender $(n)$} \\
\hline Female, $n(\%)$ & $80(40.8)$ & $40(41.7)$ & $40(40.0)$ \\
\hline Male, $n(\%)$ & $116(59.2)$ & $56(58.3)$ & $60(60.0)$ \\
\hline \multicolumn{4}{|l|}{ Age (years) } \\
\hline Mean & 46.4 & 45.9 & 46.8 \\
\hline Median (p25, p75) & $48.5(38,59)$ & $49.5(35.8,60)$ & $48(39,56.5)$ \\
\hline Minimum & 8 & 9 & 8 \\
\hline Maximum & 85 & 85 & 82 \\
\hline \multicolumn{4}{|l|}{ Missing MI } \\
\hline Patients with at least one missing MI, $n(\%)$ & $30(15.3)$ & $30(31.3)$ & $0(0.0)$ \\
\hline \multicolumn{4}{|l|}{ Per tooth, $n(\%)$} \\
\hline $11, n(\%)$ & $10(5.1)$ & $10(10.4)$ & $0(0.0)$ \\
\hline $12, n(\%)$ & $12(6.1)$ & $12(12.5)$ & $0(0.0)$ \\
\hline $21, n(\%)$ & $7(3.6)$ & $7(7.3)$ & $0(0.0)$ \\
\hline $22, n(\%)$ & $11(5.6)$ & $11(11.5)$ & $0(0.0)$ \\
\hline \multicolumn{4}{|l|}{ Endodontic treatment in $\mathrm{MI}$} \\
\hline $\begin{array}{l}\text { Patients with at least one endodontic } \\
\text { treatment in MI, } n(\%)\end{array}$ & $92(46.9)$ & $92(95.8)$ & $0(0.0)$ \\
\hline \multicolumn{4}{|l|}{ Per tooth, $n(\%)$} \\
\hline $11, n(\%)$ & $58(29.6)$ & $58(60.4)$ & $0(0.0)$ \\
\hline $12, n(\%)$ & $29(14.8)$ & $29(30.2)$ & $0(0.0)$ \\
\hline $21, n(\%)$ & $50(25.5)$ & $50(52.1)$ & $0(0.0)$ \\
\hline $22, n(\%)$ & $23(11.7)$ & $23(24.0)$ & $0(0.0)$ \\
\hline \multicolumn{4}{|l|}{ Type of NC $(n)$} \\
\hline Single canal, $n(\%)$ & $77(39.3)$ & $37(38.5)$ & $40(40.0)$ \\
\hline Two parallel canals, $n(\%)$ & $5(2.6)$ & $2(2.1)$ & $3(3.0)$ \\
\hline Y type canal, $n(\%)$ & $114(58.2)$ & $57(59.4)$ & $57(57.0)$ \\
\hline \multicolumn{4}{|l|}{ Number of nasal foramina } \\
\hline One, $n(\%)$ & $77(39.3)$ & $37(38.4)$ & $40(40.0)$ \\
\hline Two, $n(\%)$ & $107(54.6)$ & $52(54.2)$ & $55(55.0)$ \\
\hline Three or more, $n(\%)$ & $12(6.1)$ & $7(7.3)$ & $5(5.0)$ \\
\hline
\end{tabular}

$M I$ maxillary incisor, $N C$ nasopalatine canal
Measurements of the $\mathrm{NC}$ dimensions are presented in Table 2. The dimension of the foramen of Stensen (no. 1) and the length of the NC (no. 4) did not show any significant difference between dentoalveolar injury and control group. The dimension of the incisive foramen was significantly different between the groups when measured in the sagittal plane (no. 2, antero-posterior, $p=0.02$ ) but not in the axial plane (no. 5 , mesio-distal, $p=0.07$ ). Similarly, the diameter of $\mathrm{NC}$ at midway showed a significant difference between both groups when measured in the sagittal (no. 3, antero-posterior, $p=0.04$ ) but not when measured in the axial plane (no. 6, mesio-distal, $p=0.10$; Table 2). Analysis of a subgroup including dentoalveolar injury cases $(n=88)$ with any trauma signs (missing tooth or root canal filling) in the central MI only showed a significant difference $(p=0.01)$ compared to the control group for dimension no. 2 and a borderline significance $(p=0.04)$ for the dimension nos. 3, 5, and 6 .

Among all 196 cases, $22 \mathrm{NC}$ were identified with a bulging sign (11.2 \%; Table 2). Nineteen cases with a bulging signs were found in the dentoalveolar injury group, only three in the control group. This difference was statistically significant $(p=0.001)$. Mean antero-posterior, mesio-distal, and craniocaudal measurements of the bulging sign in the different CBCT planes are shown in Table 2.

Among the 744 MI present, an apical osteolysis was identified in $38(5.1 \%)$ cases in the CBCT scans. The distribution of the affected MI is shown in Table 3. Twelve periapical osteolyses did show a fusion with the $\mathrm{NC}$ in $\mathrm{CBCT}$, while for 26 periapical osteolyses, no fusion with the $\mathrm{NC}$ could be identified. This difference was statistically significant 
Table 2 Evaluation of the dimensions (mean values and standard deviation in $\mathrm{mm}$ ) of the nasopalatine canal and of a bulging sign (mean values and standard deviation in $\mathrm{mm}$ ) in the trauma and the control group

\begin{tabular}{lllll}
\hline & All & Dentoalveolar injury group & Control group & $p$ value \\
\hline Measurements & & & & \\
No. 1, mean (SD) & $2.99(1.27)$ & $3.13(1.42)$ & $2.86(1.10)$ & 0.32 \\
No. 2, mean (SD) & $3.41(1.12)$ & $3.56(1.00)$ & $3.27(1.20)$ & $\mathbf{0 . 0 2}$ \\
No. 3, mean (SD) & $2.43(1.19)$ & $2.60(1.28)$ & $2.26(1.08)$ & $\mathbf{0 . 0 4}$ \\
No. 4, mean (SD) & $8.66(2.11)$ & $8.50(2.32)$ & $8.81(1.89)$ & 0.17 \\
No. 5, mean (SD) & $3.84(1.18)$ & $3.97(1.11)$ & $3.71(1.23)$ & 0.07 \\
No. 6, mean (SD) & $3.43(1.62)$ & $3.65(1.79)$ & $3.21(1.41)$ & 0.10 \\
Bulging sign & & & & $3(3.0)$ \\
Patients, $n$ (\%) & $22(11.2)$ & $19(19.8)$ & & $\mathbf{0 . 0 0 1}$ \\
Measurements & & & $4.43(0.45)$ & 0.32 \\
Antero-posterior in SP, mean (SD) & $5.83(2.13)$ & $6.04(2.22)$ & $5.77(1.47)$ & 0.26 \\
Mesio-distal in AP, mean (SD) & $7.33(2.29)$ & $7.58(2.32)$ & $4.50(0.30)$ & 0.09 \\
Cranio-caudal in CP, mean (SD) & $6.45(2.12)$ & $6.76(2.12)$ & & \\
\hline
\end{tabular}

Bold settings indicate statistically significant findings

$S D$ standard deviation, $S P$ sagittal plane, $A P$ axial plane, $C P$ coronal plane
( $p=0.03$ ). Only two cases of residual osteolysis (after extraction and apical of a dental implant) could be found in an edentulous zone. Bucco-palatal and mesio-distal measurements of the osteolysis were significantly larger when a fusion had occurred than without ( $p=0.004, p<0.0001$; Table 3$)$.

\section{Discussion}

The present radiological study evaluated a potential difference in the size and shape of the NC between individuals having had a dentoalveolar injury with the loss of at least one MI or an endodontic treatment in an MI and those without known history of dental trauma. To the best of our knowledge, this is the first time a study correlated the history of dentoalveolar injury with morphological characteristics of the NC.

Regarding the evaluation of dimensions of the $\mathrm{NC}$ on 3D radiologic imaging (CT or CBCT), most studies measured the diameter of the openings, i.e., nasal (foramina of Stensen) and palatal (incisive foramen) $[6,11,12,14,21]$, but a comparison between studies is difficult, because the methods of measurements differ, i.e., some groups did use an oblique measurement for the palatal foramen while others performed a horizontal measurement on CBCT. Furthermore, most studies did only measure these foramina in the sagittal and not in other planes, i.e., axial. This may explain dimensional values of the incisive foramen in the present study in a range similar to those reported by Thakur et al. [22] (mean $\pm \mathrm{SD}=3.62 \mathrm{~mm} \pm$ 0.77) but smaller than in Bornstein et al. [12] (mean $\pm \mathrm{SD}=$
Table 3 Evaluation of osteolytic lesions associated with maxillary incisors with and without fusion with the nasopalatine canal

\begin{tabular}{|c|c|c|c|c|}
\hline & All & Fusion with NC & No fusion with $\mathrm{NC}$ & $p$ value \\
\hline \multicolumn{5}{|l|}{ Periapical osteolysis } \\
\hline Patients with at least one affected MI, $n$ teeth & 38 & 12 & 26 & 0.03 \\
\hline MI $11, n$ teeth & 19 & 6 & 13 & 0.17 \\
\hline MI $12, n$ teeth & 8 & 2 & 6 & 0.29 \\
\hline MI 21, $n$ teeth & 17 & 7 & 10 & 0.63 \\
\hline MI 22, $n$ teeth & 6 & 0 & 6 & 0.03 \\
\hline \multicolumn{5}{|l|}{ Other osteolysis anterior maxilla } \\
\hline Residual after extraction, $n$ & 1 & 0 & 1 & \\
\hline Apical of implant, $n$ & 1 & 0 & 1 & \\
\hline \multicolumn{5}{|l|}{ Measurements $(n)$ of osteolysis } \\
\hline Bucco-palatinal, mean (SD) & $5.45(2.22)$ & $6.76(2.13)$ & $4.79(1.93)$ & 0.004 \\
\hline Mesio-distal, mean (SD) & $6.11(3.08)$ & $8.58(2.09)$ & $4.95(2.70)$ & $<0.0001$ \\
\hline Cranio-caudal, mean (SD) & $6.57(2.72)$ & $7.52(1.86)$ & $6.03(2.89)$ & 0.053 \\
\hline
\end{tabular}

Bold settings indicate statistically significant findings

MI maxillary incisor 
$4.45 \mathrm{~mm} \pm 0.15)$, Etoz and Sisman [14] (mean $\pm \mathrm{SD}=5.06 \mathrm{~mm}$ \pm 1.48 ), and Acar and Kamburoglu [21] (mean \pm SD for females is $4.85 \mathrm{~mm} \pm 1.41$ and for males is $5.26 \mathrm{~mm} \pm 1.51)$.

The NC is subject to morphological variability regarding its type and number of nasal foramina among subjects. These variations have for the first time been emphasized in the literature by Mraiwa et al. [11] due to the availability of 3D radiographic analysis. The authors also pointed out the clinical importance of this anatomical structure particularly when planning surgical procedures such as dental implant placement. In our cohort, the most common type of canal was the Y type (58.2 \%), followed by the single canal (39.3\%), and this was similar in both the trauma and the control group, indicating that the type of canal may not be influenced by external factors. Previous studies have also identified the Y type canal as the most common (65\% by Mraiwa et al. [11], $46 \%$ by Fernandez-Alonso et al. [13]).

It has been shown by Acar and Kamburoglu [21] that the absence of a single or both central MI had a significant effect $(p<0.001)$ on the diameter of the incisive canal. The mean diameter of the incisive foramen was smaller in cases were one MI $(3.80 \mathrm{~mm})$ or both MI $(3.79 \mathrm{~mm})$ were absent, than when both were present $(3.96 \mathrm{~mm})$. In contrast, Mardinger and coworkers [22] reported an increase of the canal diameter and foramina (foramina of Stensen and incisive foramen) with the degree of ridge resorption accompanied by edentulism. In the study by Bornstein et al. [12], the absence of the central incisors only revealed a statistically significant effect on the crestal measurements of the buccal bone plate but not on the $\mathrm{NC}$ diameter. The authors reported that the longer the time period since loss of one or both central incisors was, the thinner the corresponding buccal bone wall. In the present study, the dimension of the incisive foramen was smaller in the control than in the dentoalveolar injury group, including cases with missing central MI $(n=17)$. The influence of a missing tooth on the NC diameter could have compensated the effect of the dental trauma in these cases.

Some previous studies describe the NCs based on their shape $[7,13,14,23]$. As denominations for the different variations of shape are not uniform in the literature, we did not use those in the present study. However, we checked for any local enlargement in the course of the canal and defined it as a bulging sign (Figs. 2 and 3). The bulging sign has to be differed from a bigger bulky enlargement suspicious for a NPDC [24, 25], even if the transition may be fluent and some borderline cases may be difficult to allocate to the presence of a NPDC or an enlarged NC based on analysis of CBCT scans only. The bulging sign has not been described in earlier studies, but the funnel shape cases presented by Thakur et al. [23] showed similarities to our cases with a bulging sign. This group encountered the funnel shape in $11 \%$, without any significant difference for age or gender. This is almost identical to the frequency of a bulging sign found in our cohort (11.2\%).
The significantly higher presence of the bulging sign and the borderline significance regarding larger $\mathrm{NC}$ diameters at midway level in the dentoalveolar injury compared to the control group in the present study might give a hint that a trauma could be responsible for some local NC enlargement. This tendency was enhanced when only the cases with involvement of central MI were considered in the dentoalveolar injury group (subgroup analysis). However, it is not possible to predict what will happen to a $\mathrm{NC}$ with a bulging sign, i.e., if it stays stable over time or will progress to an established NPDC. Further investigations on bulging signs in similar populations (dentoalveolar injury/non-trauma) ideally including histopathology would be interesting for future research.

The NC has been found to be at a mean distance of $3.05 \mathrm{~mm}$ from the cemento-enamel junction of central MI and at a mean distance of $5.22 \mathrm{~mm}$ from the apex of central MI in 60 men and 60 women [26]. Thus, an apical osteolysis with a bucco-palatal dimension under $5 \mathrm{~mm}$ is less likely to fuse with the NC, than if the dimension is more extended. This was confirmed by our data, showing a mean bucco-palatal dimension of $4.79 \mathrm{~mm}$ (26 patients) with an apical osteolysis without fusion and of $6.76 \mathrm{~mm}$ for apical lesions (12 patients) with fusion.

With a CBCT image, a fusion of the apical osteolysis with the $\mathrm{NC}$ can be recognized clearly as the area of interest can be evaluated in mesio-distal and orofacial directions. This can be helpful during the diagnostic procedure and also for choosing the adequate treatment protocol. Furthermore, the diagnostic accuracy to detect an apical osteolysis is better for CBCT compared to periapical radiography [27]. But the benefit and inherent risk to increase diagnostic precision and quality of treatment by using 3D imaging with $\mathrm{CBCT}$ have to be evaluated in each single case to justify higher radiation doses [28]. If using CBCT for assessment of dentoalveolar injury, a small FOV and - if possible - low-dose protocols should be selected to minimize radiation dose and exposure [29, 30]. Moreover, it has to be emphasized here that a single periapical radiograph with a significant lower radiation dose than a CBCT scan may also be sufficient for diagnosis [30].

If an apical radiolucency fuses with the NC, this corresponds biologically to an inflammatory process that has reached physiological structures within the NC. It has been described that inflammatory cells and fibroblasts of an apical granuloma release interleukins, prostaglandins, tumor necrosis factor, and growth factors that can activate epithelial cell rests to proliferate [31]. A stimulation of resting epithelial cells from the degenerated nasopalatine duct has been described previously as possible etiologic factor to initiate the formation of a NPDC $[18,19]$. It can be speculated that inflammatory mediators from apical lesions are possibly affecting these resting cells, but further research is needed to prove this hypothesis. Recently, the cellular lining of NPDCs was evaluated immunohistochemically for the first time, as well as 
mandibular radicular cysts and specimens of patients with maxillary sinusitis as a control [20]. In $56 \%$ of NPDCs, roots of teeth were not associated, while a close relationship was described in $44 \%$. The immunohistochemical findings confirmed that the NPDC lining is basically stratified squamous epithelium. It was speculated that NPDCs may arise from epithelial rest of Malassez, similar to radicular cysts. The authors concluded that some NPDCs may be inflammatory and similar to radicular cysts or even true radicular cysts located in the median maxilla and having involved the $\mathrm{NC}$ but up to now have been diagnosed as NPDCs due to current classification. In our cohort, 12 out of 196 patients had a periapical osteolysis in MI with fusion with the NC.

\section{Conclusion}

Wider dimensions of the $\mathrm{NC}$ and the presence of a bulging sign may be associated with dentoalveolar injury involving MI. Periapical osteolyses of central MI over $5 \mathrm{~mm}$ in diameter tend to fuse with the NC. Especially in cases where the lesions expand towards the palate, a fusion with the $\mathrm{NC}$ may be possible and could induce a NPDC. Clinically, in such cases, conventional and surgical treatment of the root canal treated teeth becomes more complex and may benefit from preoperative analysis using CBCT. Further research studying the NC dimension on populations with dental trauma and/or apical periodontitis of MI would be of interest to corroborate the hypothesis that NPDC can either be initiated by inflammatory processes in the neighboring teeth or sometimes stem directly from epithelial rests of Malassez. Furthermore, the long-term behavior of a bulging sign and any changes of the shape of the $\mathrm{NC}$ after fusion with an apical lesion should be examined in future research.

Acknowledgments The authors thank Mr. Lukas Martig, Institute of Mathematical Statistics and Actuarial Science, University of Bern, for his assistance during the statistical analysis and Mrs. Bernadette Rawyler, medical illustrator, School of Dental Medicine, University of Bern, for the preparation of Figs. 1, 2, and 4.

Ethical approval The study protocol was submitted and reviewed by the local ethical committee of the University Hospitals Leuven (S57587) but was exempt from formal approval due to its retrospective nature.

Conflict of interest The authors declare that they have no competing interests.

\section{References}

1. Kaste LM, Gift HC, Bhat M, Swango PA (1996) Prevalence of incisor trauma in persons 6-50 years of age: United States, 19881991. J Dent Res 75(Spec Iss):696-705
2. Andreasen JO, Borum MK, Jacobsen HL, Andreasen FM (1995) Replantation of 400 avulsed permanent incisors. 1. Diagnosis of healing complications. Endod Dent Traumatol 11:51-58

3. Hermann NV, Lauridsen E, Ahrensburg SS, Gerds TA, Andreasen JO (2012) Periodontal healing complications following extrusive and lateral luxation in the permanent dentition: a longitudinal cohort study. Dent Traumatol 28:394-402

4. Andersson L, Andreasen JO, Day P, Heithersay G, Trope M, Diangelis AJ, Kenny DJ, Sigurdsson A, Bourguignon C, Flores MT, Hicks ML, Lenzi AR, Malmgren B, Moule AJ, Tsukiboshi M, International Association of Dental Traumatology (2012) International Association of Dental Traumatology guidelines for the management of traumatic dental injuries: 2. Avulsion of permanent teeth. Dent Traumatol 28:88-96

5. Di Angelis AJ, Andreasen JO, Ebeleseder KA, Kenny DJ, Trope M, Sigurdsson A, Andersson L, Bourguignon C, Flores MT, Hicks ML, Lenzi AR, Malmgren B, Moule AJ, Pohl Y, Tsukiboshi M, International Association of Dental Traumatology (2012) International Association of Dental Traumatology guidelines for the management of traumatic dental injuries: 1. Fractures and luxations of permanent teeth. Dent Traumatol 28:2-12

6. Liang X, Jacobs R, Martens W, Hu Y, Adriaensens P, Quirynen M, Lambrichts I (2009) Macro- and micro-anatomical, histological and computed tomography scan characterization of the nasopalatine canal. J Clin Periodontol 36:598-603

7. Song WC, Jo DI, Lee JY, Kim JN, Hur MS, Hu KS, Kim HJ, Shin C, Koh KS (2009) Microanatomy of the incisive canal using threedimensional reconstruction of microCT images: an ex vivo study. Oral Surg Oral Med Oral Pathol Oral Radiol Endod 108:583-590

8. de Oliveira-Santos C, Souza PH, de Azambuja B-CS, Stinkens L, Moyaert K, Rubira-Bullen IR, Jacobs R (2012) Assessment of variations of the mandibular canal through cone beam computed tomography. Clin Oral Investig 16:387-393

9. de Oliveira-Santos C, Rubira-Bullen IR, Monteiro SA, León JE, Jacobs R (2013) Neurovascular anatomical variations in the anterior palate observed on CBCT images. Clin Oral Implants Res 24:10441048

10. von Arx T, Fodich I, Bornstein MM (2014) Proximity of premolar roots to maxillary sinus: a radiographic survey using cone-beam computed tomography. J Endod 40:1541-1548

11. Mraiwa N, Jacobs R, Van Cleynenbreugel J, Sanderink G, Schutyser F, Suetens P, van Steenberghe D, Quirynen M (2004) The nasopalatine canal revisited using 2D and 3D CT imaging. Dentomaxillofac Radiol 33:396-402

12. Bornstein MM, Balsiger R, Sendi P, von Arx T (2011) Morphology of the nasopalatine canal and dental implant surgery: a radiographic analysis of 100 consecutive patients using limited cone-beam computed tomography. Clin Oral Implants Res 22:295-301

13. Fernández-Alonso A, Suárez-Quintanilla JA, Muinelo-Lorenzo J, Bornstein MM, Blanco-Carrión A, Suárez-Cunqueiro MM (2014) Three-dimensional study of nasopalatine canal morphology: a descriptive retrospective analysis using cone-beam computed tomography. Surg Radiol Anat 36:895-905

14. Etoz M, Sisman Y (2014) Evaluation of the nasopalatine canal and variations with cone-beam computed tomography. Surg Radiol Anat 36:805-812

15. Meghji S, Qureshi W, Henderson B, Harris M (1996) The role of endotoxin and cytokines in the pathogenesis of odontogenic cysts. Arch Oral Biol 41:523-531

16. Main DM (1970) Epithelial jaw cysts: a clinicopathological reappraisal. Br J Oral Surg 8:114-125

17. Allard RH, van der Kwast WA, van der Waal I (1981) Nasopalatine duct cyst. Review of the literature and report of 22 cases. Int J Oral Surg 10:447-461

18. Abrams AM, Howell FV, Bullock WK (1963) Nasopalatine cysts. Oral Surg Oral Med Oral Pathol 16:306-332 
19. Mealey BL, Rasch MS, Braun JC, Fowler CB (1993) Incisive canal cysts related to periodontal osseous defects: case reports. J Periodontol 64:571-574

20. Tsuneki M, Maruyama S, Yamazaki M, Abé T, Adeola HA, Cheng J, Nishiyama H, Hayashi T, Kobayashi T, Takagi R, Funayama A, Saito C, Saku T (2013) Inflammatory histopathogenesis of nasopalatine duct cyst: a clinicopathological study of 41 cases. Oral Dis 19:415-424

21. Acar B, Kamburoğlu K (2015) Morphological and volumetric evaluation of the nasopalatinal canal in a Turkish population using cone-beam computed tomography. Surg Radiol Anat 37:259-265

22. Mardinger O, Namani-Sadan N, Chaushu G, Schwartz-Arad D (2008) Morphologic changes of the nasopalatine canal related to dental implantation: a radiologic study in different degrees of absorbed maxillae. J Periodontol 79:1659-62

23. Thakur AR, Burde K, Guttal K, Naikmasur VG (2013) Anatomy and morphology of the nasopalatine canal using cone-beam computed tomography. Imaging Sci Dent 43:273-281

24. Suter VG, Sendi P, Reichart PA, Bornstein MM (2011) The nasopalatine duct cyst: an analysis of the relation between clinical symptoms, cyst dimensions, and involvement of neighboring anatomical structures using cone beam computed tomography. J Oral Maxillofac Surg 69:2595-2603

25. Suter VGA, Warnakulasuriya S, Reichart PA, Bornstein MM (2015) Radiographic volume analysis as a novel tool to determine nasopalatine duct cyst dimensions and its association with presenting symptoms and postoperative complications. Clin Oral Invest. doi:10.1007/s00784-014-1391-2

26. Chatriyanuyoke P, Lu CI, Suzuki Y, Lozada JL, Rungcharassaeng K, Kan JY, Goodacre CJ (2012) Nasopalatine canal position relative to the maxillary central incisors: a cone beam computed tomography assessment. J Oral Implantol 38:713-717

27. Bornstein MM, Lauber R, Sendi P, von Arx T (2011) Comparison of periapical radiography and limited cone-beam computed tomography in mandibular molars for analysis of anatomical landmarks before apical surgery. J Endod 37:151-157

28. European Commission. Radiation Protection No 172. Cone beam CT for dental and maxillofacial radiology. Evidence-based guidelines (2012). http://www.sedentexct.eu/files/radiation protection 172.pdf [accessed on May 12, 2015]

29. Hedesiu M, Baciut M, Baciut G, Nackaerts O, Jacobs R, SEDENTEXCT Consortium (2012) Comparison of cone beam CT device and field of view for the detection of simulated periapical bone lesions. Dentomaxillofac Radiol 41:548-552

30. Pauwels R. Cone beam CT for dental and maxillofacial imaging: dose matters (2015). Radiat Prot Dosimetry 165:156-161

31. Lin LM, Huang GT, Rosenberg PA (2007) Proliferation of epithelial cell rests, formation of apical cysts, and regression of apical cysts after periapical wound healing. J Endod 33:908-916 